\title{
O impacto das percepções de justiça organizacional e da resiliência sobre o engajamento no trabalho
}

\author{
The impact of organizational justice perceptions \\ and resilience on the work engagement
}

\author{
Deanne de Freitas OLIVEIRA ${ }^{1}$ \\ Maria Cristina FERREIRA ${ }^{1}$
}

\begin{abstract}
Resumo
A presente pesquisa investigou o impacto das percepções de justiça organizacional (distributiva, processual, interpessoal e informacional) e da resiliência sobre o engajamento no trabalho, mediante um estudo de levantamento de natureza transversal. A amostra foi composta por 435 profissionais de ambos os gêneros, provenientes de organizações públicas, privadas e do terceiro setor, situadas, predominantemente, no Estado do Rio de Janeiro. A coleta de dados efetivou-se por meio de versões brasileiras da Escala de Percepção de Justiça Organizacional de Colquitt, da Escala de Resiliência de Connor-Davidson e da Escala de Engajamento no Trabalho de Utrecht. A análise de regressão múltipla linear padrão evidenciou que as percepções de justiça distributiva e interpessoal, bem como a resiliência, predisseram positivamente o engajamento no trabalho. Concluiu-se que a justiça organizacional e a resiliência consistem recursos motivacionais que promovem o engajamento no trabalho.
\end{abstract}

Palavras-chave: Justiça; Psicologia positiva; Resiliência; Trabalho.

\begin{abstract}
The aim of this study was to investigate the impact of organizational justice (distributive, procedural, interpersonal, and informational) perceptions and resilience on work engagement using a cross-sectional analysis. The sample was composed of 435 professionals, of both genders, who work in public, private, or third sector organizations based mainly in the Brazilian state of Rio de Janeiro. Data was collected using Brazilian versions of the Colquitt's Organizational Justice Perception Scale, Connor-Davidson Resilience Scale, and Utrecht Work Engagement Scale. The multiple regression analysis showed that the perceptions of distributive and interpersonal justice, as well as resilience, positively predicted work engagement. It was concluded that organizational justice and resilience are motivational resources that foster work engagement.
\end{abstract}

Keywords: Justice; Positive psychology; Resilience; Work.

O advento da Psicologia Positiva fez com que a Psicologia Organizacional, que tradicionalmente se dedicava, sobretudo, ao estudo de fenômenos negativos, como o estresse e o burnout, passasse a se interessar também e com mais frequência pelos fenômenos positivos, como a qualidade de vida e o

\footnotetext{
$\boldsymbol{\nabla} \boldsymbol{\nabla} \mathbf{v}$
}

1 Universidade Salgado de Oliveira, Programa de Pós-Graduação em Psicologia. R. Marechal Deodoro, 211 , Bloco C, $2^{\circ}$ andar, Centro, 24030-060, Niterói, RJ, Brasil. Correspondência para/Correspondence to: M.C. FERREIRA. E-mail: <mcris@centroin.com.br>. 
bem-estar no trabalho. É na esteira dessas mudanças que surge o interesse pelo estudo do engajamento no trabalho, o qual consiste em uma energia positiva, que desperta no indivíduo a vontade genuína de contribuir para o trabalho e para as atividades extra papel necessárias à sua organização (Albrecht, 2010).

Os estudos sobre o engajamento no trabalho ainda são poucos no exterior, e praticamente inexistentes no Brasil, conforme levantamento realizado nas bases de dados Psychinfo, Scientific Eletronic Library Online (SciELO) e Portal de Periódicos Eletrônicos de Psicologia (PePSIC), no mês de agosto de 2014. Nesse sentido, o estudo do referido fenômeno se reveste de importância teórica e prática, na medida em que a análise da rede nomológica que o compõe está apenas se iniciando, além de que indivíduos engajados trazem várias consequências positivas para suas organizações empregadoras. Fundamentando-se nessas considerações, o presente trabalho teve como objetivo geral investigar o impacto das percepções de justiça organizacional e da resiliência sobre o engajamento no trabalho.

\section{Engajamento no trabalho}

O engajamento pode ser descrito por três dimensões: vigor, dedicação e absorção (Schaufeli \& Bakker, 2004). O vigor caracteriza-se por níveis elevados de energia e pela persistência, mesmo frente a dificuldades. A dedicação, por sua vez, refere-se às sensações de significado, entusiasmo, inspiração, orgulho e desafio despertados pelo trabalho. A absorção, por fim, associa-se à plena concentração e dedicação ao próprio trabalho, fazendo com que o indivíduo tenha dificuldades de se separar do mesmo.

O principal modelo teórico desenvolvido para a explicação do engajamento no trabalho é o de demandas e recursos do trabalho (Bakker \& Demerouti, 2008). As demandas associam-se a aspectos do contexto do trabalho que exigem esforços fisiológicos e psicológicos do indivíduo e podem acabar por sobrecarregá-lo, levando-o a um estado de

748 fadiga o qual esgota toda a sua energia. Estão dire- tamente relacionadas a problemas de saúde no trabalho, como, por exemplo, o estresse e o burnout.

Já os recursos dizem respeito a fatores motivacionais intrínsecos (autonomia e feedback, por exemplo) e extrínsecos (suporte de colegas e de supervisores, clareza de papéis e participação na tomada de decisões, por exemplo) os quais permitem ao indivíduo satisfazer suas necessidades psicológicas e atingir suas metas de trabalho (Bakker \& Leiter, 2010). Esses fatores influenciam diretamente o engajamento no trabalho (Hakanen \& Roodt, 2010).

O modelo de demandas e recursos preconiza, ainda, que recursos pessoais também interferem no engajamento no trabalho. Esses recursos consistem em autoavaliações positivas associadas à capacidade de controlar o próprio ambiente de forma mais eficaz, levando o indivíduo a se tornar intrinsecamente mais motivado a atingir suas metas e, consequentemente, obter altos padrões de desempenho e de satisfação no trabalho. Tomando por base o modelo de demandas e recursos, o presente estudo optou por investigar o papel desempenhado por um recurso do trabalho, quais sejam as percepções de justiça organizacional, e um recurso pessoal, qual seja a resiliência, sobre o engajamento de trabalhadores brasileiros.

\section{Justiça organizacional e engajamento no trabalho}

A justiça organizacional decorre das relações entre trabalhador e organização, as quais podem levar à ativação de mecanismos de defesa e recusa ou a atitudes e comportamentos de envolvimento e disponibilidade, dentre outros (Greenberg, 1987). A primeira dimensão de justiça estudada foi a distributiva, que adota como regra básica o princípio da equidade. Diz respeito, portanto, à justiça dos resultados alcançados, ao modo como foi feita a distribuição dos recursos positivos ou negativos, como salários, promoções, serviços etc. (Assmar, Ferreira, \& Souto, 2005).

A pesquisa em justiça ganha novo desdobramento com Thibaut e Walker (1975), que introduzem na literatura sociopsicológica a dimensão de justiça processual (processo pelo qual os resultados são atribuídos) (Mendonça \& Mendes, 2005). 
Caberá a Bies e Moag (1986) introduzir a dimensão da justiça interacional, a qual se refere à qualidade do tratamento (modo polido e claro) que as pessoas recebem no momento da aplicação dos processos de distribuiç̧ão.

Colquitt, Conlon, Wesson, Porter e Ng (2001) defendem, ainda, que a justiça interacional se subdivide em justiça interpessoal e informacional. A primeira reflete o grau de dignidade e respeito com os quais as pessoas são tratadas por autoridades ou outras partes envolvidas em executar procedimentos ou determinar resultados. A segunda focaliza-se nas explicações dadas às pessoas responsáveis por transmitir informações sobre os motivos de alguns procedimentos serem usados de uma determinada forma ou de alguns resultados serem distribuídos de uma certa maneira.

O modelo tetra-dimensional de justiça vem sendo adotado em pesquisas recentes (Akremi, Vandenberghe, \& Camerman, 2010; Cole, Bernerth, Walter, \& Holt, 2010), muito embora alguns autores (Cojuharenko \& Patient, 2013) ainda prefiram utilizar o modelo tri-dimensional, no qual a justiça interpessoal e a informacional são agrupadas na dimensão de justiça interacional. No entanto, a decomposição dessa dimensão permite identificar com maior clareza os efeitos separados da justiça interpessoal e da informacional nas atitudes e sentimentos dos membros organizacionais (Colquitt, 2012), como é o caso, por exemplo, do engajamento no trabalho. Apoiando-se nesses pressupostos, a presente investigação adotou o modelo de Colquitt et al. (2001), com o objetivo de identificar o impacto das quatro dimensões de justiça sobre o engajamento no trabalho.

Os estudos sobre as relações entre as percepções de justiça e o comprometimento organizacional afetivo, construto correlato ao engajamento no trabalho, vêm demonstrando que as percepções de justiça distributiva, processual e interpessoal associam-se positivamente ao comprometimento organizacional afetivo (Ferreira et al., 2006; Rego, 2002; Rego \& Souto, 2004). Tem-se verificado, também, que as percepções de justiça distributiva e processual predizem o engajamento laboral (He, Zhu, \& Zheng, 2014; Saks, 2006; Strom, Sears, \& Kelly, 2014). Fundamentando-se nesse referencial, foi formulada a Hipótese 1: as percepções de justiça distributiva, processual, interpessoal e informacional predizem positivamente o engajamento no trabalho.

\section{Resiliência e engajamento no trabalho}

No contexto da Psicologia, a resiliência possui diversos significados, que incluem a recuperação de experiências traumáticas, a superação de desvantagens para vencer na vida e a resistência ao estresse para o adequado funcionamento nas tarefas do dia a dia (Ungar, Dumont, \& McDonald, 2005). No ambiente organizacional, essa característica diz respeito à capacidade do indivíduo resistir a situações de intensa pressão mediante o uso de recursos adaptativos capazes de manter uma relação saudável entre ele e seu trabalho (Barlach, Limongi, \& Malvezzi, 2008).

Os autores que vêm abordando a resiliência dividem-se entre os que acreditam ser ela um traço pessoal e hereditário e os que acreditam ser um processo aprendido ao longo do desenvolvimento. Os defensores da resiliência como um traço individual consideram que as pessoas resilientes, quando expostas à adversidade e aos riscos da pobreza, apresentam uma capacidade intrínseca de adaptação positiva às condições de vida adversa, as quais independem do contexto em que se encontram (Melillo \& Ojeda, 2005).

A concepção da resiliência como um processo enfatiza que ela é aprendida na interação recíproca dos seres humanos com a adversidade e na adaptação positiva daí resultante. Considera-se, assim, que o processo de resiliência ocorre quando o indivíduo não demonstra sinais de desajuste, apesar da exposição à adversidade, revelando, assim, uma adaptação positiva ou resiliente (Melillo \& Ojeda, 2005).

De particular importância para os objetivos do presente trabalho é a resiliência que os empregados apresentam frente às adversidades do contexto laboral. Sob essa perspectiva, ela foi concebida como uma característica ou processo que leva os trabalhadores a apresentarem respostas diferen- 
ciadas aos desafios e pressões vivenciados em seu ambiente de trabalho.

Os estudos sobre resiliência e engajamento no trabalho ainda são incipientes. Nas bases de dados pesquisadas, foi localizada uma única investigação com foco nessa relação, a qual constatou que baixos resultados em resiliência associavam-se a menores resultados em engajamento (Carvalho, Calvo, Martín, Campos, \& Castilho, 2006). Já o estudo de Avey, Wernsing e Luthans (2008) concluiu que o capital psicológico, o qual tem a resiliência como uma de suas dimensões, constituía-se em um preditor positivo do engajamento no trabalho. Apoiando-se nessas considerações, foi formulada a Hipótese 2: a resiliência prediz positivamente 0 engajamento no trabalho.

\section{Método}

\section{Participantes}

O estudo consistiu em um levantamento de natureza transversal, conduzido no período de junho a setembro de 2012, por meio de um processo de amostragem por conveniência. O número mínimo de participantes para atender aos pressupostos da análise de regressão múltipla deve ser, segundo Tabachnick e Fidell (2007), de $\mathrm{N} \geq 50$ + 8n (n igual ao número de variáveis antecedentes), o que equivaleria a um número mínimo de 90 participantes; ou, de acordo com Hair, Andersen, Tatham e Black (2005), igual a 50 vezes o número de variáveis independentes envolvidas na investigação, o que corresponderia, nesta pesquisa, a um total mínimo de 250 casos. O presente estudo, porém, contou com um total de 435 participantes, número bem acima do mínimo requerido em ambos os critérios.

A amostra ficou, portanto, composta por 435 trabalhadores, membros de diferentes organizações públicas $(23,6 \%)$, privadas $(69,9 \%)$ ou do terceiro setor $(6,5 \%)$, situadas predominantemente no estado do Rio de Janeiro e atuando principalmente no setor de serviços (95,9\%). Desse total, $71,7 \%$ foram do gênero feminino e suas idades
Desvio-Padrão - $D P=10,66)$. Com relação à escolaridade, a maioria dos respondentes $(73,2 \%)$ possuía nível superior. O tempo de trabalho desses indivíduos nas organizações pesquisadas variou de 1 a 38 anos $(M=6,39 ; D P=7,03)$, enquanto seu tempo total de serviço variou de 1 a 43 anos $(M=12,71$; $D P=9,37)$, sendo que $15,5 \%$ ocupavam cargos gerenciais e $26,4 \%$ trabalhavam em mais de uma organização.

\section{Instrumentos}

A justiça organizacional foi avaliada pela Escala de Percepção de Justiça de Colquitt (2001), composta por 20 itens em formato de resposta tipo Likert de cinco pontos (variando de "nunca" a "sempre"), distribuídos por quatro dimensões: justiça distributiva (4 itens), justiça processual (7 itens), justiça interpessoal (5 itens) e justiça informacional (4 itens). A referida escala foi adaptada e validada em amostras brasileiras no estudo de Assmar et al. (2002). Na presente pesquisa, os índices de consistência interna, calculados pelo Alfa de Cronbach, foram iguais a 0,91 (justiça distributiva); 0,87 (justiça processual); 0,82 (justiça interpessoal); e 0,87 (justiça informacional).

A resiliência foi avaliada pela Escala de Resiliência de Connor-Davidson (CD-RISC, Connor-Davidson Resilience Scale), de autoria de Connor e Davidson (2003), na versão reduzida desenvolvida por Campbell-Sills e Stein (2007). A escala é unifatorial, sendo composta por 10 itens (CD-RISC - 10) em formato de resposta tipo Likert de cinco pontos (variando de "nem um pouco verdadeiro" a "quase sempre verdadeiro"). A versão brasileira da escala foi validada por Lopes e Martins (2011). Na atual investigação, o coeficiente Alfa de Cronbach da escala foi igual a 0,83.

O engajamento no trabalho foi avaliado pela versão reduzida da Escala de Engajamento no Trabalho de Utrecht (UWES, Utrecht Work Engagement Scale), desenvolvida por Schaufeli e Bakker (2003) e validada em amostras brasileiras por Valentini e Ferreira (2014). A escala se compõe de 9 itens em formato de resposta tipo Likert de cinco pontos, variando de "nunca" a "diariamente". Neste estu- 
do, o Alpha de Cronbach da escala foi igual a 0,91. Incluiu-se, ainda, no instrumento de coleta de dados, perguntas destinadas à coleta de informações sociodemográficas sobre os participantes e o Termo de Consentimento Livre e Esclarecido.

\section{Procedimentos}

Inicialmente, o projeto foi submetido e aprovado pelo Comitê de Ética em Pesquisa da instituição dos autores (Protocolo n 46/2012, aprovado em 1 de maio de 2012). Em seguida, foram feitos contatos com os trabalhadores de diferentes organizações, mediante visitas a seus locais de trabalho e por e-mail. Os que concordaram voluntariamente em participar da pesquisa responderam em lápis e papel aos instrumentos, em tempo livre, e o devolveram imediatamente após seu preenchimento. Os que foram contatados por e-mail, receberam um link com a página na qual o questionário se encontrava, preencheram-no eletronicamente e o enviaram aos pesquisadores também por meio eletrônico. As coletas foram realizadas nas duas formas (impressa e on-line) com o intuito de se garantir um número adequado de participantes o mais brevemente possível. O anonimato do indivíduo e da organização foi assegurado a todos os envolvidos na investigação.

\section{Resultados}

Inicialmente, foram realizados testes $t$ para amostras independentes com o intuito de se verificar as diferenças porventura existentes entre as médias das distintas escalas compreendidas pela investigação no grupo de participantes que responderam aos questionários na versão impressa $(73,6 \%)$ e na versão on-line (26,4\%). Os dados obtidos revelaram, no entanto, que não houve diferenças significativas entre as médias dos dois grupos em nenhuma das cinco escalas.

As médias e os desvios-padrão de cada uma dessas escalas, bem como os coeficientes de correlação entre elas, encontram-se na Tabela 1. Considerando-se que todas as escalas eram de cinco pontos (ponto médio de 3), foi possível verificar que as médias de todas elas situaram-se acima do ponto médio. As maiores médias foram obtidas nas escalas de justiça distributiva $(3,97)$, de engajamento $(3,82)$ e de justiça processual $(3,60)$. No que diz respeito às correlações entre a variável dependente e as variáveis independentes, verificou-se que o engajamento no trabalho apresentou, segundo os critérios de Miles e Shevlin (2001), correlações positivas e significativas moderadas com a resiliência, com as percepções de justiça processual, com as de justiça interpessoal e com as de justiça informacional; além de ter obtido uma correlação significativa positiva e elevada com as percepções de justiça distributiva.

O exame dos pressupostos necessários à realização de análises de regressão foi feito de acordo com as recomendações de Tabachnick e Fidel (2007). No exame da homocedasticidade, linearidade e normalidade das distribuições das variáveis, foram adotados métodos estatísticos (cálculo de assimetria e curtose) e gráficos (histogramas com sobreposição da curva normal e gráficos de dispersão dos resíduos), tendo-se concluído pela não neces-

Tabela 1

Médias, desvios-padrão e coeficientes de correlação entre as percepções de justiça, resiliência e engajamento

\begin{tabular}{lccccccc}
\hline Escalas & $M$ & $D P$ & 1 & 2 & 3 & 4 & 5 \\
\hline Resiliência (1) & 3,27 & 0,83 & - & - & - & - & - \\
Justiça Processual (2) & 3,81 & 0,94 & $0,30^{*}$ & - & - & - & - \\
Justiça Distributiva (3) & 3,97 & 0,82 & $0,30^{*}$ & $0,50^{*}$ & - & - & - \\
Justiça Interpessoal (4) & 3,76 & 0,94 & $0,23^{*}$ & $0,41^{*}$ & $0,29^{*}$ & - & - \\
Justiça Informacional (5) & 3,78 & 0,65 & $0,18^{*}$ & $0,46^{*}$ & $0,36^{*}$ & $0,67^{*}$ & - \\
Engajamento (6) & 3,82 & 0,88 & $0,44^{*}$ & $0,43^{*}$ & $0,55^{*}$ & $0,41^{*}$ & $0,38^{*}$ \\
\hline
\end{tabular}

Nota: ${ }^{*} p<0,01$

M: Média; DP: Desvio-Padrão. 
sidade de transformação de qualquer uma das variáveis do estudo.

No que diz respeito à multicolinearidade, constatou-se que nenhuma das correlações entre as variáveis preditoras foi maior que 0,90 , além dos valores de tolerância estarem acima de 0,20 e os valores do Fator de Inflação da Variância (VIF, Variance Inflation Factor), abaixo de 4. Não foi detectada a presença de dados atípicos (outliers) univariados (os valores de $z$ de todas as escalas foram abaixo de 3,29; $p<0,001$ ) ou multivariados (a distância de Cook em nenhum caso foi maior que o limite máximo de 1) que justificassem a remoção de casos da amostra.

Com o objetivo de se verificar o poder preditivo das percepções de justiça (distributiva, processual, interpessoal e informacional) e da resiliência sobre o engajamento no trabalho, foi realizada uma análise de regressão múltipla linear padrão, na qual o modelo retido revelou-se significativo e explicou $45 \%$ da variância da variável critério $\left(R^{2}=0,45\right.$; $F[5,361]=60,65 ; p<0,001)$. No entanto, somente as percepções de justiça distributiva, a resiliência e as percepções de justiça interpessoal se constituíram em preditores positivos e significativos do engajamento no trabalho (Tabela 2), com a justiça distributiva destacando-se no modelo como a variável de maior poder preditivo.

\section{Discussão}

A presente pesquisa teve como objetivo geral investigar a influência das percepções de quatro dimensões de justiça organizacional (distributiva, processual, interpessoal, informacional) e da resiliência sobre o engajamento no trabalho. Os dados

Tabela 2 obtidos evidenciaram que, dentre as percepções das quatro dimensões de justiça organizacional, somente as de justiça distributiva e de justiça interpessoal constituíram-se preditoras positivas e significativas do engajamento no trabalho, sendo que a primeira destacou-se como a principal preditora da variável critério.

Esses resultados confirmam parcialmente a Hipótese 1, segundo a qual as percepções de justiça distributiva, processual, interpessoal e informacional seriam preditoras do engajamento. Eles divergem, porém, do estudo de Saks (2006), que observou uma associação positiva e significativa apenas entre a justiça processual e o engajamento no trabalho. Além disso, se mostram incongruentes com as investigações as quais apontam para o fato de que a justiça processual tem primazia sobre as demais dimensões de justiça, enquanto preditora de atitudes e comportamentos positivos no trabalho (Ferreira et al., 2006; Rego, 2002; Rego \& Souto, 2004).

O fato da justiça distributiva ter apresentado poder preditivo bem maior que a interpessoal pode ser visto como uma evidência de que, na amostra pesquisada, a adoção de distribuições justas de recursos constituiu-se em prática mais eficaz para a obtenção do engajamento no trabalho quando comparada à utilização de um tratamento digno e respeitoso com os empregados. Em outras palavras, as práticas de justiça estrutural (recompensas recebidas) tiveram primazia sobre as práticas de justiça social (tratamento recebido) na configuração do engajamento no trabalho.

Tomados em conjunto, porém, os resultados sobre a influência das percepções de justiça sobre o engajamento podem ser interpretados a partir da teoria da troca social. Segundo essa teoria, um

Regressão múltipla linear padrão das percepções de justiça e da resiliência sobre o engajamento no trabalho

\begin{tabular}{lccc}
\hline Variáveis preditoras & $\beta$ & $t$ & $p$ \\
\hline Resiliência & 0,24 & 5,78 & 0,001 \\
Justiça processual & 0,04 & 0,92 & 0,360 \\
Justiça distributiva & 0,38 & 8,32 & 0,001 \\
Justiça interpessoal & 0,21 & 3,97 & 0,001 \\
Justiça informacional & 0,06 & 1,14 & 0,254 \\
\hline
\end{tabular}


tratamento justo por parte da organização configura-se como uma troca social à qual o empregado responde apresentando comportamentos organizacionais positivos (Siqueira, Gomide Júnior, \& Oliveira, 2002), como é o caso do engajamento no trabalho.

De acordo com a Hipótese 2, a resiliência iria predizer positivamente o engajamento no trabalho, o que foi totalmente confirmado. Esse achado vai ao encontro dos estudos anteriores de Carvalho et al. (2006), no qual se verificou que baixos resultados em resiliência se associavam a menores resultados em engajamento, e da pesquisa de Avey et al. (2008), na qual se concluiu que o capital psicológico, o qual tem a resiliência como uma de suas dimensões, se constituía em um preditor positivo do engajamento no trabalho.

A concepção de resiliência adotada no estudo foi a de que ela consiste em uma característica ou processo o qual permite a superação dos desafios e pressões vivenciados no ambiente de trabalho. Seria de se esperar que os sujeitos dotados de maior resiliência se mostrassem propensos a um engajamento mais efetivo no trabalho, devido ao fato de apresentarem maior capacidade de superar as dificuldades nele vivenciadas e, consequentemente, respondendo a elas de forma mais positiva, como de fato foi constatado.

Cumpre ressaltar, ainda, que na atual investigação, o engajamento foi predito não apenas por características do contexto do trabalho (percepções de justiça distributiva e interpessoal), mas também por características individuais (resiliência), muito embora o poder preditivo do contexto do trabalho tenha sido maior quando comparado ao fator individual. Esse resultado está alinhado aos autores (Rupp, 2011) que defendem a realização de investigações as quais utilizem conjuntamente fatores contextuais e individuais como antecedentes das atitudes e comportamentos que ocorrem no ambiente organizacional, como forma de se obter maior compreensão desses fenômenos.

Outra interpretação possível para as atuais evidências pode ser feita recorrendo-se ao modelo de demandas e recursos do trabalho (Bakker \& Demerouti, 2008). Segundo esse modelo, os recursos do trabalho permitem o alcance de metas de trabalho e o crescimento pessoal. Podem ser extrínsecos ou intrínsecos. Os extrínsecos caracterizam-se como aspectos do contexto laboral os quais estimulam o desejo de se dedicar às tarefas, o que facilita o alcance das metas do trabalho. Os recursos intrínsecos, por sua vez, satisfazem a necessidades humanas básicas.

Nesse sentido, a justiça distributiva, ao prover o empregado com recompensas, pode ser considerada como um recurso do trabalho que desempenha um papel motivacional extrínseco, estimulando, assim, o engajamento. A justiça interpessoal, contudo, pode ser vista como um recurso de natureza motivacional intrínseca, na medida em que o tratamento polido do supervisor contribui para a satisfação de necessidades de relacionamento interpessoal e, em consequência, provoca o engajamento no trabalho.

O modelo de demandas e recursos (Bakker \& Demerouti, 2008) preconiza ainda que os recursos pessoais também interferem no engajamento no ambiente laboral. Estes consistem em autoavaliações positivas associadas à capacidade de controlar o próprio ambiente de forma mais eficaz e que levam os indivíduos a se tornarem intrinsecamente mais motivados a atingir suas metas.

Nesse sentido, a resiliência adequa-se totalmente à conceituação de recurso pessoal, em função de permitir ao indivíduo um estilo ativo de enfrentamento que o ajuda a controlar seu ambiente de trabalho e a obter sucesso. Em síntese, os dados ora obtidos podem ser tomados como uma extensão das pressuposições do modelo de demandas e recursos do trabalho a amostras brasileiras.

Esses resultados permitem a elaboração de sugestões de estratégias a serem implementadas no futuro, como forma de reforçar os níveis de engajamento no ambiente laboral. A adoção de sistemas de recompensas pautados em distribuições justas, bem como o treinamento de líderes voltados à aquisição de habilidades interpessoais poderão, assim, se revelar eficazes para a melhoria do engajamento no trabalho dos membros organizacionais.

No que tange às limitações da presente pesquisa, o fato dela ter sido conduzida em grande 
parte apenas no estado do Rio de Janeiro diminui suas possibilidades de generalização. Por outro lado, a amostra foi voluntária, razão pela qual não foi possível controlar se apenas os indivíduos mais ou menos engajados em seu trabalho ou com percepções de justiça mais ou menos elevadas participaram da pesquisa. Cumpre registrar, por fim, que o fato de terem sido utilizadas duas formas de coleta de dados (impressa e on-line) não trouxe nenhum impacto aos resultados da pesquisa, conforme evidenciado pelos resultados dos testes $t$ realizados entre os dois grupos nas diferentes escalas do estudo.

\section{Considerações Finais}

Em relação a uma agenda futura de pesquisas, sugere-se a realização de estudos longitudinais que possam aprofundar a compreensão dos mecanismos pelos quais as percepções de justiça impactam o engajamento no trabalho. Outrossim, a condução de investigações acerca de outros preditores do engajamento no ambiente laboral em amostras específicas de profissionais também poderá fornecer novo lume ao conjunto de fatores os quais caracterizam a rede nomológica do engajamento do trabalho em organizações brasileiras. Os dados ora obtidos permitem concluir que o presente trabalho reveste-se de importância teórica e prática, em função de ter proposto e testado variáveis ainda pouco exploradas em amostras brasileiras. Complementarmente, avançou na explicação dos fatores que concorrem para o engajamento laboral.

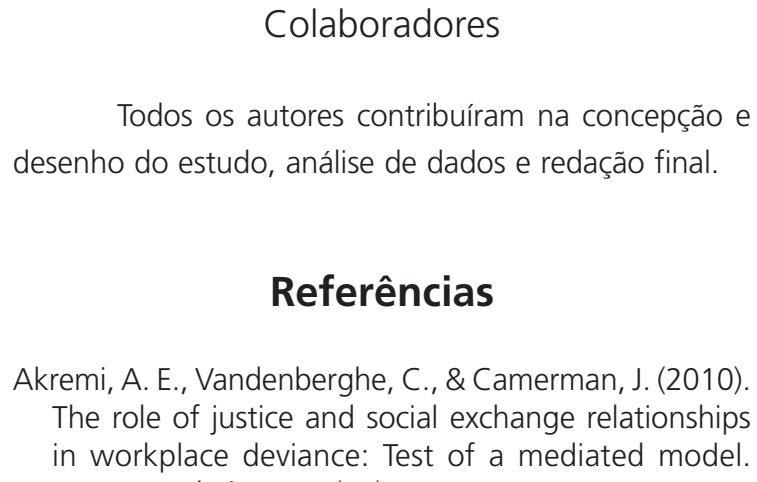

\section{Referências}

Akremi, A. E., Vandenberghe, C., \& Camerman, J. (2010). The role of justice and social exchange relationships in workplace deviance: Test of a mediated model.

Albrecht, S. L. (2010). Employee engagement: 10 key questions for research and practice. In S. L. Albrecht (Ed.), Handbook of employee engagement: Perspectives, issues, research and practice (pp.3-19). Northampton: Edward Elgar.

Assmar, E. M. L., Ferreira, M. C., \& Souto, S. O. (2005). Justiça organizacional: uma revisão crítica da literatura. Psicologia: Reflexão e Crítica, 18(3), 443-453.

Assmar, E. M. L., Ferreira, M. C., Souto, S. O., Souza, A. L. R., Mac-Cord, F., \& Borges, D. (2002). Justiça organizacional: um modelo multidimensional para uso no Brasil. Resumos do I Congresso Psicologia e Profissão, São Paulo, SP. Recuperado em maio 15, 2012, de http:// www.cienciaeprofissao.com.br/congre/downloads.asp

Avey, J. B., Wernsing, T. S., \& Luthans, F. (2008). Can positive employees help positive organizational change? Impact of psychological capital and emotions on relevant attitudes and behaviors. Journal of Applied Behavioral Science, 44(1), 48-70.

Bakker, A. B., \& Demerouti, E. (2008). Towards a model of work engagement. Career Development International, 13(3), 209-223.

Bakker, A., \& Leiter, M. P. (2010). Work engagement: Introduction. In A. B. Bakker \& M. P. Leiter (Eds.), Work engagement: A handbook of essential theory and research (pp.1-9). New York: Psychology Press.

Barlach, L., Limongi, A. C., \& Malvezzi, F. S. (2008). O conceito de resiliência aplicado ao trabalho nas organizações. Revista Interamericana de Psicologia, 42(1), 101-112.

Bies, R. J., \& Moag, J. S. (1986). Interactional justice: Communication criteria of fairness. In R. J. Lewicki, B. H. Sheppard, \& M. H. Bazerman (Eds.), Research on negotiation in organizations (pp.43-55). Greenwich: JAl Press.

Campbell-Sills, L., \& Stein, M. B. (2007). Psychometric analysis and refinement of the Connor-Davidson Resilience Scale (CD-RISC): Validation of a 10-Item measure of resilience. Journal of Traumatic Stress, 20(6), 1019-1028.

Carvalho, V. A. M. L., Calvo, B. F., Martín, L. H., Campos, F. R., \& Castilho, I. C. (2006). Resiliencia y el modelo burnout-engagement en cuidadores formales de ancianos. Psicothema, 18(4), 791-796.

Cojuharenco, I., \& Patient, D. (2013). Workplace fairness versus unfairness: Examining the differential salience of facets of organizational justice. Journal of Occupational and Organizational Psychology, 86(3), 371-393.

Cole, M. S., Bernerth, J. B., Walter, F., \& Holt, D. T. (2010). Organizational justice and individuals' withdrawal: Unlocking the influence of emotional exhaustion. Journal of Management, 47(3), 367-390.

Colquitt, J. A. (2001). On the multidimensionality of organizational justice: A construct validation of a measure. Journal of Applied Psychology, 86(3), 386-400. 
Colquitt, J. A. (2012). Organizational justice. In S. W. J. Kozlowski (Ed.), The Oxford handbook of organizational psychology (Vol.1, pp.526-547). New York: Oxford University Press.

Colquitt, J. A., Conlon, D. E., Wesson, M. J., Porter, C. O. L. H., \& Ng, K. Y. (2001). Justice at the millennium: A meta-analytic review of 25 years of organizational justice research. Journal of Applied Psychology, 86(3), 425-445.

Connor, K. M., \& Davidson, J. R. T. (2003). Development of a new resilience scale: The Connor-Davidson Resilience Scale (CD-RISC). Depression and Anxiety, 18(2), 76-82.

Ferreira, M. C., Assmar, E. M. L., Souto, S. O., Omar, A. G., Delgado, H. U., González, A. T., \& Galáz, M. M. F. (2006). Individualismo e coletivismo, percepções de justiça e comprometimento em organizações latino-americanas. Revista Interamericana de Psicologia, 40(1), 13-24.

Greenberg, J. (1987). A taxonomy of organizational justice theories. Academy of Management Review, 12(1), 9-22.

Hair, J. F., Andersen, R. E., Tatham, R. L., \& Black, W. C. (2005). Análise multivariada de dados. Porto Alegre: Artmed.

Hakanen, J. J., \& Roodt, G. (2010). Using the job demands-resources model to predict engagement: Analysing a conceptual model. In A. B. Bakker \& M. P. Leiter (Eds.), Work engagement: $A$ handbook of essential theory and research (pp.85-101). New York: Psychology Press.

He, H., Zhu, W., \& Zheng, X. (2014). Procedural justice and employee engagement: Roles of organizational identification and moral identity centrality. Journal of Business Ethics, 122(4), 681-695.

Lopes, V. R., \& Martins, M. C. F. (2011). Validação fatorial da escala de resiliência de Connor-Davidson (CD-RISC-10) para brasileiros. Psicologia: Organizações e Trabalho, $11(2), 36-50$.

Melillo, A., \& Ojeda, E. N. S. (2005). Resiliência: descobrindo as próprias fortalezas. Porto Alegre: Artmed.

Mendonça, H., \& Mendes, A. M. (2005). Experiências de injustiça, sofrimento e retaliação no contexto de uma organização pública do estado de Goiás. Psicologia em Estudo, 10(3), 489-498.

Miles, J. N. V., \& Shevlin, M. E. (2001). Applying regression and correlates: A guide for students and researchers. London: Sage.
Rego, A. (2002). Comprometimento afetivo dos membros organizacionais: O papel das percepções de justiça. Revista de Administração Contemporânea, 6(2), 209-241.

Rego, A., \& Souto, S. (2004). A percepção de justiça como antecedente do comprometimento organizacional: um estudo luso-brasileiro. Revista de Administração Contemporânea, 8(1), 151-177.

Rupp, D. E. (2011). An employee-centered model of organizational justice and social responsibility. Organizational Psychology Review, 1(1), 72-94.

Saks, A. M. (2006). Antecedents and consequences of employee engagement. Journal of Managerial Psychology, 21(7), 600-619.

Schaufeli, W. B., \& Bakker, A. B. (2003). UWES: Utrecht Work Engagement Scale: Preliminary manual. Utrecht: Occupational Health Psychology Unit.

Schaufeli, W. B., \& Bakker, A. B. (2004). Job demands, job resources, and their relationship with burnout and engagement: A multi-sample study. Journal of Organizational Behavior, 25(3), 293-315.

Siqueira, M. M., Gomide Júnior, S., \& Oliveira, A. F. (2002). Cidadania, justiça e cultura nas organizações: estudos psicossociais. São Paulo: Universidade Metodista.

Strom, D. L., Sears, K. L., \& Kelly, K. M. (2014). Work engagement: The roles of organizational justice and leadership style in predicting engagement among employees. Journal of Leadership and Organizational Studies, 21(1), 71-82.

Tabachnik, B. G., \& Fidell, L. (2007). Using multivariate statistics ( $5^{\mathrm{a}}$ ed.). Boston: Pearson.

Thibaut, J. W., \& Walker, L. (1975). Procedural justice: A psychological analysis. Hillsdale: Lawrence Erlbaum Associates.

Ungar, M., Dumont, C., \& McDonald, W. (2005). Risk, resilience and outdoor programming for at-risk children. Journal of Social Work, 5(3), 319-338.

Valentini, F., \& Ferreira, M. C. (2014). Brazilian confirmatory factor analysis of the Utrecht Work Engagament Scale. Book of Abstracts of The $9^{\text {th }}$ Conference of the International Test Comission, San Sebastian, Espanha. Retrieved August 5, 2014, from http://www.itc 2014ss.com/uploads/Libro\%20grab\%20CD\%200 ng.pdf

Recebido: janeiro 31, 2014

Versão final: agosto 20, 2014

Aprovado: setembro 19, 2014 
